\title{
On composite types of tunnel number two knots
}

\author{
by \\ Kanji Morimoto \\ Department of IS and Mathematics, Konan University \\ Okamoto 8-9-1, Higashi-Nada, Kobe 658-8501, Japan \\ morimoto@konan-u.ac.jp
}

\begin{abstract}
Let $K$ be a tunnel number two knot. Then, by considering the $(g, b)$ decompositions, $K$ is one of $(3,0)-,(2,1)-,(1,2)$ - or $(0,3)$-knots. In the present paper, we analyze the connected sum summands of composite tunnel number two knots and give a complete table of those summands from the point of view of $(g, b)$-decompositions.
\end{abstract}

Keywords: $(g, b)$-decompositions, tunnel number two knots, connected sum

2010 Mathematics Subject Classification : 57M25, 57M27

\section{Introduction}

Let $K$ be a knot in $S^{3}$. Then it is well known that $K$ can be uniquely decomposed into finitely many prime knots, which is due to Schubert ([11]) and is called the prime decomposition of $K$. Consider the tunnel number of $K$ denoted by $t(K)$, where the tunnel number is the minimal number of arcs properly embedded in the knot exterior $E(K)$ whose complementary space is homeomorphic to a handlebody. By the definition of the tunnel number, we have $t(K)=g(E(K))-1$, where $g(E(K))$ is the Heegaard genus of $E(K)$. In the present paper, we analyze the prime decomposition of tunnel number two knots and give a complete table from the point of view of $(g, b)$-decompositions defined as below.

Let $g$ and $b$ be non-negative integers with $(g, b) \neq(0,0)$. Then we say that a knot $K$ has a $(g, b)$-decomposition if there is a genus $g$ Heegaard splitting $\left(V_{1}, V_{2}\right)$ of $S^{3}$ such that $K$ intersects each handlebody in a $b$-string trivial arc system. In particular, if $b=0$ then we define a $(g, 0)$-decomposition as a genus $g$ Heegaard splitting of $S^{3}$ such that at least one of the two handlebodies contains $K$ as a central loop of a handle. If $g=0$, then a $(0, b)$-decomposition is the ordinary $b$-bridge decomposition.

This decomposition is due to Doll ([1]) and is a generalization of the ordinary bridge decompositions due to Schubert ([12]). Then, by the definition and a little observation, we see that if a knot $K$ has a $(g, b)$-decomposition then $t(K) \leq g+b-1$. This concept, $(g, b)$-decomposition of knots, plays very important role from the point 
of view of the tunnel numbers and the distance due to Hempel ([2]). For example, see [3], [4] or [8].

Let $B$ be a 3 -ball and $t_{1} \cup t_{2}$ be two arcs properly embedded in $B$. Then $\left(B, t_{1} \cup t_{2}\right)$ is called a 2 -string tangle. We say that $\left(B, t_{1} \cup t_{2}\right)$ is trivial if $t_{1} \cup t_{2}$ is a 2 -string trivial arc system in $B$, that $\left(B, t_{1} \cup t_{2}\right)$ is free if $c l\left(B-N\left(t_{1} \cup t_{2}\right)\right)$ is a genus two handlebody, where $N\left(t_{1} \cup t_{2}\right)$ is a regular neighborhood of $t_{1} \cup t_{2}$ in $B$, that $\left(B, t_{1} \cup t_{2}\right)$ is essential if $c l\left(\partial B-N\left(t_{1} \cup t_{2}\right)\right)$ is incompressible in $\operatorname{cl}\left(B-N\left(t_{1} \cup t_{2}\right)\right)$ and that $t_{i}(i=1,2)$ is unknotted if $\left(B, t_{i}\right)$ is a trivial ball pair. We say that a knot $K$ has a 2 -string essential free tangle decomposition if $\left(S^{3}, K\right)$ is decomposed into a union of two 2string essential free tangles.

For a knot $K$ in $S^{3}$, we define the following two conditions $c(1)$ and $c(2)$.

$c(1):\left(S^{3}, K\right)$ has a 2 -string essential free tangle decomposition such that exactly one of the two tangles has an unknotted component.

$c(2):\left(S^{3}, K\right)$ has a 2 -string essential free tangle decomposition such that each tangle of the two tangles has an unknotted component.

Under the above notations, for composite tunnel number two knots, we have shown the following :

Theorem $1([\mathbf{5}, \mathbf{6}])$ Let $K$ be a composite tunnel number two knot, then one of the following holds.

(1) $K$ is the connected sum of a tunnel number one knot and a knot with a $(1,1)$ decomposition,

(2) $K$ is the connected sum of a 2-bridge knot and a knot with a c(1)- or a c(2)condition.

For composite knots with $(2,1)$-decompositions, we have shown the following :

Theorem 2 ([7]) Let $K$ be a composite knot with a $(2,1)$-decomposition, then one of the following holds.

(1) $K$ is the connected sum of a tunnel number one knot and a 2-bridge knot,

(2) $K$ is the connected sum of two knots with $(1,1)$-decompositions,

(3) $K$ is the connected sum of a 2-bridge knot and a knot with a c(2)-condition.

For 3-bridge knots, by the additivity of bridge indices due to Schubert, we have the following :

Theorem 3 ([12]) Let $K$ be a composite 3 -bridge knot, then $K$ is the connected sum of two 2-bridge knots.

In the present paper, for knots with (1,2)-decompositions, we will show the follow- 
ing :

Theorem 4 Let $K$ be a composite knot with a $(1,2)$-decomposition, then $K$ is the connected sum of a knot with a $(1,1)$-decomposition and a 2-bridge knot.

By the way, to state the above results more precisely, we need to define the term $(g, b)$-knots. To do this, by a little observation, we have :

Fact 1 (1) If a knot $K$ has $a(g-1, b+1)$-decomposition, then $K$ has a $(g, b)$ decomposition.

(2) If a knot $K$ has a $(g, b-1)$-decomposition, then $K$ has a $(g, b)$-decomposition.

By the above fact, in the present paper, we say that a knot $K$ is a $(g, b)$-knot if $K$ has a $(g, b)$-decomposition but has neither $(g-1, b+1)$-decomposition nor $(g, b-1)$ decomposition. In addition, we say that a knot $K$ is a $c(i)$-knot if $K$ has a $c(i)$ condition $(i=1,2)$. Then the above Theorems $1,2,3$ and 4 are rewritten as follows. We note that, since $(3,0)$-knots are tunnel number two knots which have no $(2,1)$ decompositions, it is needed to delete the $c(2)$-condition in Theorem 1 . We further note that there is no knot which has both conditions $c(1)$ and $c(2)$ because of the unique 2-string essential free decomposition theorem due to Ozawa ([10]).

Theorem 1 Let $K$ be a composite (3,0)-knot, then one of the following holds.

(1) $K$ is the connected sum of a $(2,0)$-knot and a $(1,1)$-knot,

(2) $K$ is the connected sum of a $(0,2)$-knot and a $c(1)$-knot.

Theorem 2 Let $K$ be a composite $(2,1)$-knot, then one of the following holds.

(1) $K$ is the connected sum of a $(2,0)$-knot and a $(0,2)$-knot,

(2) $K$ is the connected sum of two $(1,1)$-knots,

(3) $K$ is the connected sum of a $(0,2)$-knot and a $c(2)$-knot.

Theorem 3 Let $K$ be a composite $(0,3)$-knot, then $K$ is the connected sum of two $(0,2)$-knots.

Theorem 4 Let $K$ be a composite (1,2)-knot, then $K$ is the connected sum of a $(1,1)$-knot and $a(0,2)$-knot.

Then, by summarizing the above results, we have the following table of composite tunnel number two knots from the point of view of $(g, b)$-decompositions. 


\begin{tabular}{|l|l|}
\hline$(g, b)$ & composite types \\
\hline$(3,0)$ & $(2,0) \#(1,1)$ or $(0,2) \# c(1)$ \\
\hline$(2,1)$ & $(2,0) \#(0,2), \quad(1,1) \#(1,1) \quad$ or $(0,2) \# c(2)$ \\
\hline$(1,2)$ & $(1,1) \#(0,2)$ \\
\hline$(0,3)$ & $(0,2) \#(0,2)$ \\
\hline
\end{tabular}

Table 1: Composite types of tunnel number two knots

Remark 1 Concerning the $c(1)$ - and $c(2)$-conditions, we have the following :

(1) If a knot $K$ has $c(1)$-condition then $K$ is a prime $(3,0)$-knot. Hence $(0,2) \# c(1)$ is included in $(0,2) \#(3,0)$ and this is the tunnel number degeneration " $2+1=2$ ".

(2) If a knot $K$ has $c(2)$-condition then $K$ is a prime $(2,1)$-knot. Hence $(0,2) \# c(2)$ is included in $(0,2) \#(2,1)$ and this is also the tunnel number degeneration " $2+1=2$ ".

Remark 2 By Fact 1, the family of knots with $(g, b)$-decompositions contains the family of knots with $(g-1, b+1)$-decompositions and the family of knots with $(g, b-1)$ decompositions. Hence we have the $(g, b)$-diagram as illustrated in Figure 1.

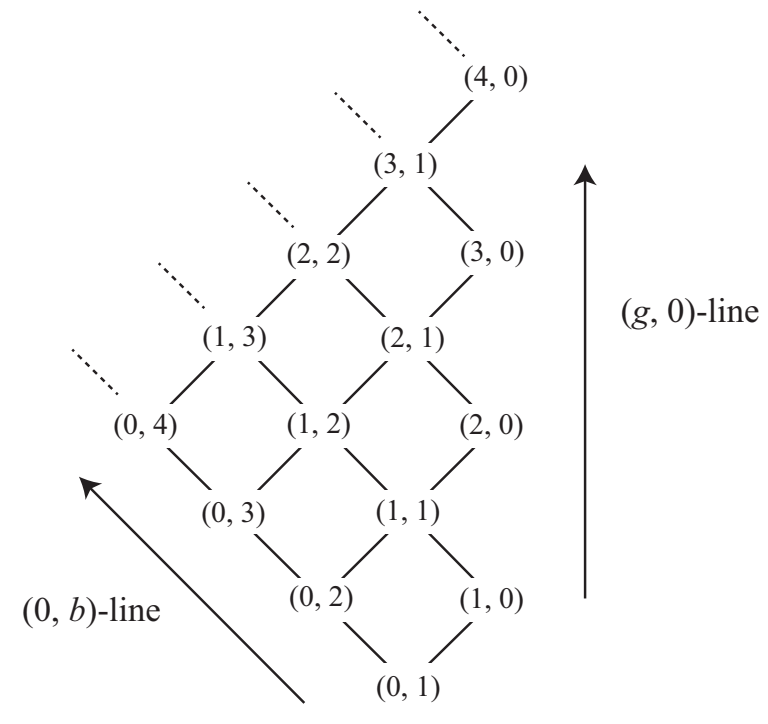

Figure 1: The $(g, b)$-diagram

\section{Proof of Theorem 4}

Let $K$ be a composite knot in $S^{3}$ with the decomposing 2-sphere $S$. Suppose $K$ has a $(1,2)$-decomposition. Then there is a genus one Heegaard splitting $\left(V_{1}, V_{2}\right)$ of $S^{3}$ such that $K$ intersects $V_{i}(i=, 2)$ in 2-string trivial arc system, where $V_{i}$ is a solid 
torus.

Put $V_{i} \cap K=\gamma_{i}^{1} \cup \gamma_{i}^{2}$, and $S_{i}=V_{i} \cap S$. Then, by taking a spine of $V_{1}$, we may assume that $S_{1}$ consists of disks not intersecting $\gamma_{1}^{1} \cup \gamma_{1}^{2}$ and $S_{2}$ is a planar surface properly embedded in $V_{2}$ intersecting $\gamma_{2}^{1} \cup \gamma_{2}^{2}$ in two points. In addition we may assume that the number of the components of $S_{1}$ is minimal among all decomposing 2 -spheres of $K$. Then we have :

Lemma 2.1 Each component of $S_{1}$ is one of the following three types as in Figure 2:

(1) a separating disk which cuts off a 3-ball containing one of $\gamma_{1}^{1} \cup \gamma_{1}^{2}$,

(2) a separating disk which cuts off a 3-ball containing both of $\gamma_{1}^{1} \cup \gamma_{1}^{2}$,

(3) a non-separating disk.

Proof. Let $D$ be a component of $S_{1}$. Suppose $D$ is a separating disk. Then $D$ divides $V_{1}$ into a 3 -ball and a solid torus. If the 3 -ball contains no component of $\gamma_{1}^{1} \cup \gamma_{1}^{2}$, then we can reduce the number of the components of $S_{1}$ and this contradicts the minimality. Thus the 3 -ball contains at least one component of $\gamma_{1}^{1} \cup \gamma_{1}^{2}$ and this completes the proof of the lemma.

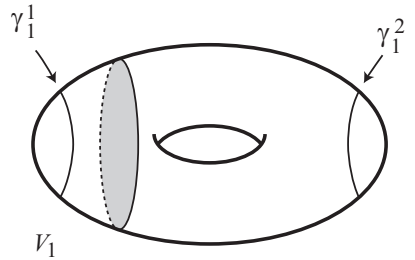

(1)

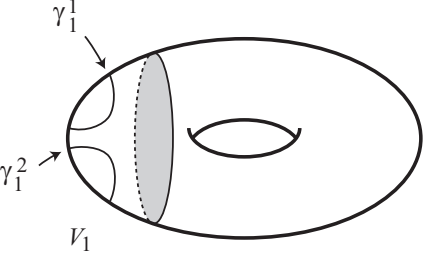

(2)

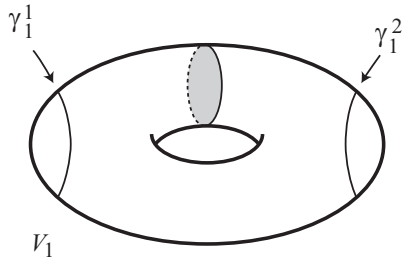

$(3)$

Figure 2: Disks in $V_{1}$

Next, let $E_{1}$ and $E_{2}$ the disks in $V_{2}$ for the triviality of $\gamma_{2}^{1}$ and $\gamma_{2}^{2}$ respectiverly, and $E_{3}$ and $E_{4}$ the two non-separating disks in $V_{2}$ such that $E_{3} \cup E_{4}$ divides $V_{2}$ into two 3-balls each of which contains one of $E_{1} \cup E_{2}$ as in Figure 3.

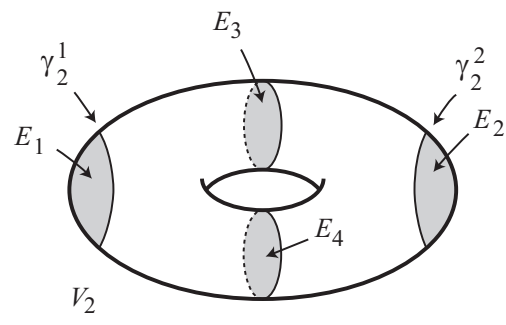

Figure 3: $E_{1} \cup E_{2} \cup E_{3} \cup E_{4}$ in $V_{2}$

Put $E=E_{1} \cup E_{2} \cup E_{3} \cup E_{4}$, then by standard cut and paste operations, we may assume that each component of $S_{2} \cap E$ is an arc properly embedded in $E$. We say that an arc $\alpha$ properly embedded in $S_{2}$ is $\gamma$-essential if $\alpha$ is essential in $S_{2}-\left(\gamma_{2}^{1} \cup \gamma_{2}^{2}\right)$. 
Suppose there is an $\operatorname{arc} \alpha$ in $S_{2} \cap E$ which is $\gamma$-essential in $S_{2}$. Let $\Delta$ be the disk in $E$ such that $\partial \Delta$ is the union of $\alpha$ and a subarc of $\partial E-\left(\gamma_{2}^{1} \cup \gamma_{2}^{2}\right)$. We may assume that $\Delta \cap S_{2}=\alpha$ by changing the disks $E$ if necessary. Then we can perform a boundary compression of $S_{2}$ at $\alpha$ along $\Delta$ from $V_{2}$ to $V_{1}$, and we get a band, say $b$, in $V_{1}$. If $b$ connects two different disks in $S_{1}$, then we can reduce the number of the components of $S_{1}$ and this contradicts the minimality. Thus $b$ connects a single disk, and the union of the band and the disk is an annulus in $V_{1}$. Then we have :

Lemma 2.2 The annulus is one of the following three types as in Figure 4 :

(1) the union of a separating disk of type (1) in Lemma 2.1 and a band which is contained in the solid torus component and winds around the longitude exactly once, (2) the union of a separating disk of type (2) in Lemma 2.1 and a band which is contained in the 3-ball component such that the compressing disk of the annulus in $\partial V_{1}$ intersects $\gamma_{1}^{1} \cup \gamma_{1}^{2}$ in two points.

(3) the union of a non-separating disk and a band such that the compressing disk of the annulus in $\partial V_{1}$ intersects $\gamma_{1}^{1} \cup \gamma_{1}^{2}$ in two points,

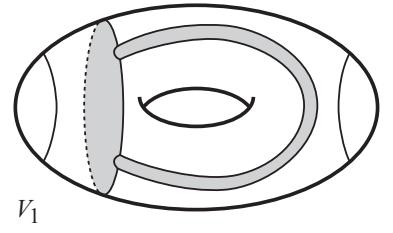

(1)

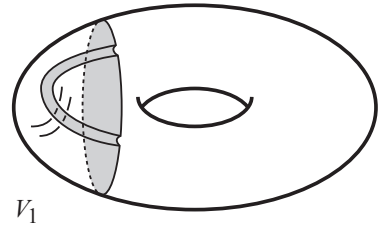

(2)

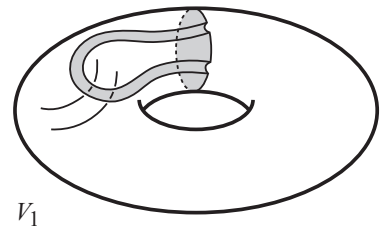

(3)

Figure 4: Annuli in $V_{1}$

Proof. Let $D$ be a disk component of $S_{1}$ which is connected to the band $b$, then $D \cup b$, say $A$, is an annulus properly embedded in $V_{1}$. Suppose $D$ is a separating disk of type (1). Then, since $D$ divides $V_{1}$ into a 3 -ball and a solid torus, $b$ is contained in the 3 -ball or in the solid torus. If $b$ is contained in the 3 -ball, then $A$ is compressible in $V_{1}$ and by standard cut and paste operation, we can take another decomposing 2-sphere which intersects $V_{1}$ in fewer disk components. This contradicts the minimality. Thus $b$ is contained in the solid torus component. Then by the same reason as above, $b$ winds around the solid torus at least once in the longitudinal direction. However, if the band winds more than once, then, since we can regard each disk of $S-A$ is a 2-handle for $V_{1}$, we have the lens space not $S^{3}$. This is a contradiction. Thus $b$ winds around $V_{1}$ exactly once and $A$ is an annulus of type (1).

Next suppose $D$ is a separating disk of type (2). Then, since $D$ divides $V_{1}$ into a 3 -ball and a solid torus, $b$ is contained in the 3 -ball or in the solid torus. If $b$ is contained in the solid torus, then by the same reason as above $b$ winds around the solid torus exactly once. However, in this case, $A$ can be pushed out into $V_{2}$ and we 
can reduce the number of the components of $S_{1}$. Thus $b$ is contained in the 3 -ball component. Then each component of $\partial A$ bounds a disk in $\partial V_{1}$. If at least one disk intersects $\gamma_{1}^{1} \cup \gamma_{1}^{2}$ in 0 or 1 point, then we can take another decomposing 2-sphere which intersects $V_{1}$ in fewer disk components. This contradicts the minimality and shows that $A$ is an annulus of type (2).

Finally suppose $D$ is a non-separating disk. Then, since $D$ cuts open $V_{1}$ into a 3-ball, $A$ is compressible in $V_{1}$. Let $\Delta$ be a compressing disk for $A$ in $\partial V_{1}$. If $\Delta \cap\left(\gamma_{1}^{1} \cup \gamma_{1}^{2}\right)=0$ or 1 point, then by cut and paste operation we can take another decomposing 2-sphere which intersects $V_{1}$ in fewer disk components. This contradicts the minimality. Thus $\Delta \cap\left(\gamma_{1}^{1} \cup \gamma_{1}^{2}\right)=2,3$ or 4 points. However, if it is 3 or 4 points, then by taking another compressing disk for $A$, i.e., a meridian disk of $V_{1}$, we can reduce the number of the components of $S_{1}$ and have a contradiction similarly. Thus $A$ is an annulus of type (3), and this completes the proof.

Let $n$ be the number of the components of $S_{1}$, then :

Lemma 2.3 We have $n=1$. Hence $S_{1}$ is a single disk not intersecting $\gamma_{1}^{1} \cup \gamma_{1}^{2}$ and $S_{2}$ is a single disk intersecting $\gamma_{2}^{1} \cup \gamma_{2}^{2}$ in two points.

Proof. Suppose $n>1$. Then, since $S_{2}$ has a non-trivial fundamental group, $S_{2} \cap E$ has a $\gamma$-essential arc. Thus we can perform a boundary compression and get a band $b_{1}$ in $V_{1}$. Then by the minimality of $S_{1}, b_{1}$ connects a single component of $S_{1}$ and an annulus is produced in $V_{1}$. Continue this procedure. Then, at some $k$ th stage, we have that $b_{1}, b_{2}, \cdots, b_{k}$ are the bands each of which connects a single component of $S_{1}$ and $b_{k+1}$ connects two different components of $S_{1}$, where to avoid the confusion of notations we use the same notations of $S_{1}$ and $S_{2}$ even after the boundary compressions. We note that there are no two bands $b_{i}$ and $b_{j}$ which connect the same disk because at each stage core arcs of those bands are $\gamma$-essential in $S_{2}$. Then, at this stage, we have $k$ annuli $A_{1}, A_{2}, \cdots, A_{k}$ in $V_{1}$.

Suppose $k<n$. Then, since there remains a disk component of $S_{1}$, we have the following two cases :

(i) $b_{k+1}$ connects a disk and an annulus $A_{i}$,

(ii) $b_{k+1}$ connects two annuli $A_{i}, A_{j}(i<j)$.

In case (i), we can use the inverse operation of boundary compression introduced by Ochiai in [9], and can reduce the number of the components of $S_{1}$. This is a contradiction. In case (ii), we have two subcases, (ii-a) : $A_{1}, A_{2}, \cdots, A_{k}$ are all mutually parallel annuli of type (1) in Lemma 2.2, (ii-b) : $A_{1}, A_{2}, \cdots, A_{k}$ are of type (2) or of type (3) in Lemma 2.2. Then, in case (ii-a), $b_{k+1}$ does not run over the band $b_{j}$. In case (ii-b), by the existence of the disk of type (3) in Lemma 2.1, we see that $b_{k+1}$ does not run over the band $b_{j}$. Then, in both cases, we can pull back the bands 
$b_{j}, \cdots, b_{k}$ leaving $b_{k+1}$ in $V_{1}$. This means that case (ii) is reduced to case (i) and we have a contradiction. Thus we have $k=n$.

By the above arguments, we can put $S_{1}=A_{1} \cup A_{2} \cup \cdots \cup A_{n}$ and $S_{2}=D_{1}^{*} \cup D_{2}^{*} \cup B_{1} \cup$ $B_{2} \cup \cdots \cup B_{n-1}$, wher $A_{i}(i=1,2, \cdots, n)$ is an annulus in $V_{1}, B_{i}(i=1,2, \cdots, n-1)$ is an annulus in $V_{2}$ and $D_{i}^{*}(i=1,2)$ is a disk in $V_{2}$ intersecting $\gamma_{2}^{1} \cup \gamma_{2}^{2}$ in a single point.

Suppose $D_{1}^{*}$ is a non-separating disk and $\partial D_{1}^{*}$ is identified with a component of $\partial A_{i}$ for some $i$. Then, since $V_{1} \cup V_{2}=S^{3}, A_{i}$ is of type (1) in Lemma 2.2. Then, by Lemma 2.2, those components of $S_{1}$ are all mutually parallel annuli of type (1). This means that each component of $\partial S_{2}$ is a meridian of $V_{2}$. However, each annulus component of $S_{2}$ has a boundary component which is not a meridian by Lemma 2.2. This is a contradiction, and shows that both of $D_{1}^{*}$ and $D_{2}^{*}$ are separating disks.

Let $X$ be a 3 -ball in $V_{2}$ cut off by $D_{1}^{*}$. Then we may assume that $X \cap S_{2}=D_{1}^{*}$, and since $D_{i}^{*} \cap\left(\gamma_{2}^{1} \cup \gamma_{2}^{2}\right)(i=1,2)$ is a single point, $\left(X \cap \partial V_{2}\right) \cap\left(\gamma_{2}^{1} \cup \gamma_{2}^{2}\right)$ is a single point or three points. However, there is no annulus component of $S_{1}$ whose boundary component bounds a disk in $\partial V_{1}$ intersecting $\gamma_{1}^{1} \cup \gamma_{1}^{2}$ in a single point or in three points. This contradiction is due to the hypothesis $n>1$, and completes the proof.

Proof of Theorem 4. By Lemma 2.3, $S_{1}$ is a single separating disk not intersecting $\gamma_{1}^{1} \cup \gamma_{1}^{2}$, and $S_{2}$ is a single separating disk intersecting $\gamma_{2}^{1} \cup \gamma_{2}^{2}$ in two points. We may assume that $S_{2} \cap E$ consists of arcs properly embedded in $E$ and that the number of the components of $S_{2} \cap E$ is minimal among all decomposing 2-spheres intersecting $V_{i}(i=1,2)$ in a single disk. We note that $S_{2} \cap E$ contains two arcs which meets a point of $S_{2} \cap\left(\gamma_{2}^{1} \cup \gamma_{2}^{2}\right)$. Then we have the following two cases :

(i) $S_{2} \cap E$ consists of exactly two arcs each of which meets a point of $S_{2} \cap\left(\gamma_{2}^{1} \cup \gamma_{2}^{2}\right)$,

(ii) $S_{2} \cap E$ contains a $\gamma$-essential arc properly embedded in $S_{2}$.

Suppose we are in case (i). Then we have an arc component $\alpha$ of $S_{2} \cap E_{1}$ which intersects $\gamma_{2}^{1}$ in a single point. Let $\Delta$ be one of the two disks in $E_{1}$ cut off by $\alpha$. Then we may assume $\Delta \cap S=\alpha$, and can isotope $S$ along the disk $\Delta$ so that $S \cap V_{i}(i=1,2)$ is a single separating disk intersecting $\gamma_{i}^{1} \cup \gamma_{i}^{2}$ in a single point as in Figure 5.

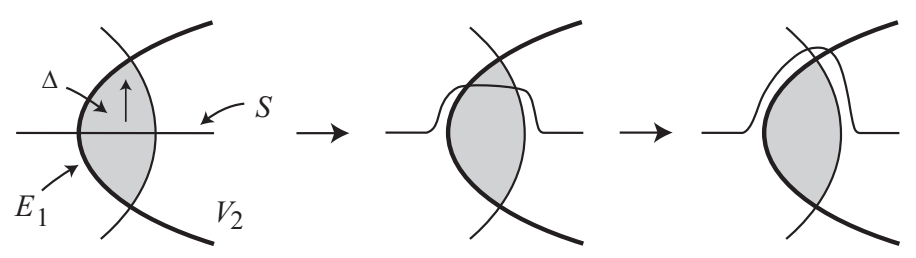

Figure 5: The isotopy along $\Delta$

Let $X_{i}(i=1,2)$ be the 3 -ball in $V_{i}$ cut off by $S_{i}$. Since, $S_{i} \cap\left(\gamma_{i}^{1} \cup \gamma_{i}^{2}\right)$ is a single point, $\left(X_{i} \cap \partial V_{i}\right) \cap\left(\gamma_{i}^{1} \cup \gamma_{i}^{2}\right)$ is one or three points. If it is one point, then the tangle 
$\left(X_{i}, X_{i} \cap\left(\gamma_{i}^{1} \cup \gamma_{i}^{2}\right)\right)$ is a trivial tangle. In this case, $\left(X_{1} \cup X_{2},\left(X_{1} \cup X_{2}\right) \cap K\right)$ is a 1-string trivial tangle and this means that $S$ bounds a trivial ball pair. This is a contradiction because $S$ is a decomposing 2-sphere of the non-trivial connected sum of $K$. Thus we have that $\left(X_{i} \cap \partial V_{i}\right) \cap\left(\gamma_{i}^{1} \cup \gamma_{i}^{2}\right)$ consists of three points as in Figure 6 .

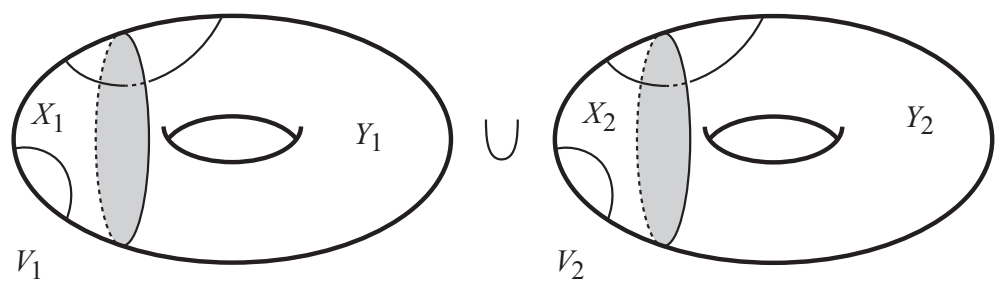

Figure 6: The intersection of $S$ and $V_{1} \cup V_{2}$ in case (i)

Let $X_{i}(i=1,2)$ be the 3 -ball in $V_{i}$ cut off by $S_{i}$ and $Y_{i}$ the solid torus in $V_{i}$ cut off by $S_{i}$. Then $\left(X_{i}, X_{i} \cap\left(\gamma_{i}^{1} \cup \gamma_{i}^{2}\right)\right)$ is a 2 -string trivial tangle and $\left(Y_{i}, Y_{i} \cap\left(\gamma_{i}^{1} \cup \gamma_{i}^{2}\right)\right)$ is a solid torus with a single trivial arc. Hence $X_{1} \cup X_{2}$ extends to a 2-bridge decomposition of a knot and $Y_{1} \cup Y_{2}$ extends to a knot with a $(1,1)$-decomposition, i.e., $K$ is the connected sum of a $(0,2)$-knot and a $(1,1)$-knot.

Next, suppose we are in case (ii). Let $\alpha$ be a $\gamma$-essential arc properly embedded in $S_{2}$. Then we may assume that $\alpha$ is outermost in $E$. Perform a boundary compression of $S_{2}$ at $\alpha$. Then, since $\alpha$ is an arc in $S_{2}$ which splits the two points $S_{2} \cap\left(\gamma_{2}^{1} \cup \gamma_{2}^{2}\right)$, we can put $S_{1}=A$ and $S_{2}=D_{1}^{*} \cup D_{2}^{*}$, where $A$ is an annulus of type (1) in Lemma 2.2 and $D_{i}^{*}(i=1,2)$ is a meridian disk of $V_{2}$ intersecting $\gamma_{2}^{1} \cup \gamma_{2}^{2}$ in a single point. We note that if $A$ is an annulus of type (2) in Lemma 2.2, then $D_{i}^{*}$ is a separating disk intersecting $\gamma_{i}^{1} \cup \gamma_{i}^{2}$ in two points and this is a contradiction.

Let $X_{i} \cup Y_{i}(i=1,2)$ be the two components cut off by $S_{i}$, where $X_{1}$ and $X_{2}$ are identified and $Y_{1}$ and $Y_{2}$ are identified as in Figure 7. Then, by adding a 2-handle to $X_{1}$ along the annulus $A,\left(X_{1}, \gamma_{1}^{1}\right)$ extends to a 2 -string trivial tangle, where a core arc of the 2-handle is regarded as a string. In addition $\left(X_{2}, X_{2} \cap\left(\gamma_{2}^{1} \cup \gamma_{2}^{2}\right)\right)$ is also a 2 -string trivial tangle.

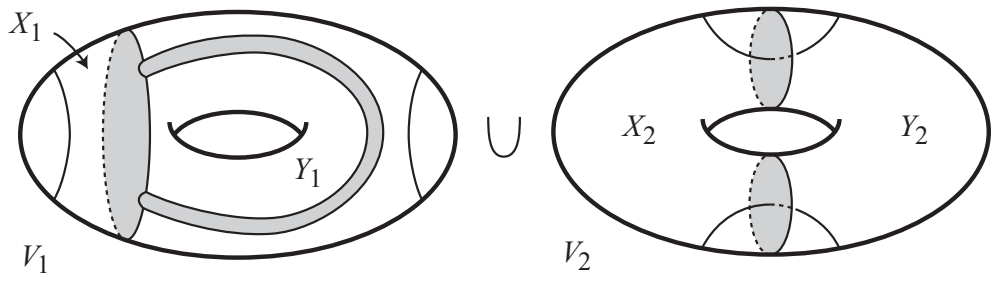

Figure 7: The intersection of $S$ and $V_{1} \cup V_{2}$ in case (ii)

Hence $X_{1} \cup X_{2}$ extends to a 2-bridge decomposition of a knot. On the other hand, $\left(Y_{1}, \gamma_{1}^{2}\right)$ is a solid torus with a trivial arc, and by adding a 1-handle to $Y_{2}$ along the 
two disks $D_{1}^{*} \cup D_{2}^{*}$, $\left(Y_{2}, Y_{2} \cap\left(\gamma_{2}^{1} \cup \gamma_{2}^{2}\right)\right)$ extends to a solid torus with a trivial arc, where a core arc of the 1-handle is regarded as a part of the trivial arc. Hence $Y_{1} \cup Y_{2}$ extends to a $(1,1)$-decomposition of a knot. This shows that $K$ is the connected sum of a $(0,2)$-knot and a $(1,1)$-knot, and completes the proof of Theorem 4.

\section{References}

[1] H. Doll, A generalized bridge number for links in 3-manifolds, Math. Ann., 294 (1992) 701-717

[2] J. Hempel, 3manifolds as viewed from the curve complex. Topology, 40 (2001) 631-657.

[3] J. Johnson and A. Thompson, On tunnel number one knots that are not $(1, n)$, J. Knot Theory Ramifications, 20 (2011) 609-615.

[4] T. Li and R. Qiu, On the degeneration of tunnel numbers under connected sum, arXiv:math/1310.5054.

[5] K. Morimoto, On the additivity of tunnel number of knots, Topology Appl., 53 (1993) 37-66.

[6] K. Morimoto, Charaterization of tunnel number two knots which have the property " $2+1=2$ ", Topology Appl., 64 (1995) 165-176.

[7] K. Morimoto, Charaterization of composite knots with 1-bridge genus two, J. Knot Theory Ramifications, 10 (2001) 823-840.

[8] Y. Minsky, Y. Moriah and S. Schleimer, High distance knots, arXiv:math/0607265.

[9] M. Ochiai, On Haken's theorem and its extension, Osaka J. Math. 20 (1983) 461-480.

[10] M. Ozawa, On uniqueness of essential tangle decompositions of knots with free tangle decompositions, Proc. Appl. Math. Workshop 8 ed. G. T. Jin and

K. H. Ko, KAIST, Taejon (1998) 227-232.

[11] H. Schubert, Die eindeutige Zerlegbarkeit eines Knoten in Primknoten, Sitzungsber. Akad. Wiss. Heidelberg, math.-nat. KI. 3 (1949) Abh: 57-104.

[12] H. Schubert, Über eine numerische Knoteninvariante, Math. Z. 61 (1954) 245288. 